Review Article

\title{
A Review of Occlusal Registration Materials Utilized in Recording Various Occlusal Relations
}

\section{Ganaraj Shetty ${ }^{1}$, Manoj Shetty ${ }^{2}$}

${ }^{1}$ Senior Lecturer, Department of Prosthodontics and Crown \& Bridge, ${ }^{2}$ Prof \& Head, Department of Implantology, A. B. Shetty M emorial Institute of Dental Sciences, Deralakatte M angalore - 575018.

Corresponding Author: Ganaraj Shetty, Senior Lecturer, Department of Prosthodontics and Crown \& Bridge, A. B. Shetty Memorial Institute of Dental Sciences, Deralakatte, Mangalore - 575018.

Received

: 12.03 .2018

Review Completed : 25.05.2018

Accepted

: 10.07.2018

Keywords : Centric relation, bite registration materials, Condylar guidance

\begin{tabular}{|c|}
\hline Access this article online \\
\hline Quick Response Code \\
\hline \\
\end{tabular}

\begin{abstract}
:
The success of any prosthetic rehabilitation depends on several factors; most important factor is precisely recording of centric relation and transferring the same to the articulator. When partial or completely edentulous arches are present, cast mounting is considerably more complex. Interocclusal records are the means by which the interarch relationships are transferred from mouth to an articulator. The purpose of this review is to evaluate the accuracy and stability of the materials used in routine clinical practice to obtain interocclusal records.
\end{abstract}

\section{Introduction}

The essentials of gnathodynamaic concepts include centric relation, anterior guidance, occlusal vertical and horizontal dimension, mandibular movements. Occlusion is the key element in all kinds of dental rehabilitation. The longevity of a restoration depends on occlusion and loading conditions. Earlier concepts of biomechanical aspects of dental occlusion have currently been combined with biological, behavioral and neuromuscular control of the stomatognathic system. ${ }^{[1]}$ Although dental occlusion has been the most questioned and complicated issue of dentistry; recording the maxillomandibular relationship by applying the basic principles and key factors bring success for the prosthetic rehabilitative treatments. ${ }^{[2]}$ The factors that affects recording of occlusal dimension, may be the neural control, centric relationship, alveolar bone resorption, postural positions, loss or attrition of natural teeth contacts that may also influence the determination of the vertical dimension. Accurate and balanced interocclusal records can be made in clinical situations using several techniques and materials, the interocclusal records minimize need for intraoral adjustments or errors in occlusal relationships during prosthesis delivery. ${ }^{[3]}$ The materials, commonly used as interocclusal recording medium are impression plaster, modelling compound, modelling wax, zincoxide eugenol impression paste, acrylic resin, elastomeric impression materials. Apart from materials, the techniques used to record centric relation is the most critical step, various methods used for recording interocclusal relationships are direct interocclusal bite records, graphic method, functional method and cephalometric analysis. ${ }^{[4 \mid[5]}$ The normal functioning of the patient's proprioception and the tactile sense is very essential in the making of a stable and accurate interocclusal record, during earlier days interocculusal record were obtained by using a direct and non-precise 
method, the centric relation record was made by placing a thermoplastic material, usually wax or impression compound, between the occlusal rims and having the patient close onto the material, this method was known as the "M ush, "Biscuit", or "Squash" Bite. Along with placing the bite registration materials in between the maxillary and mandibular arches, the direct visual perception and the tactile sense of the dentist also play a major role in making of a centric relation record. ${ }^{[5[1]][6]}$ In 1756, Phillip Pfaff, the dentist of Fredrick the Great of Germany, was the 1st to describe the technique of "taking a bite." Until the end of nineteenth century it was the most routinely used method to record centric relation. In 1954, Brown recommended repeated closure into softened wax rims. Greene had his patients hold their jaws apart for seconds to fatigue the muscles (muscle engram) ${ }^{[8] 16]}$ Gradually, these procedures evolved into interocclusal records as they are usually done today..$^{[9[10:[11]}$ The techniques and methods to record interocclusal relationships were introduced as an attempt to equalize the pressure of vertical occlusal contact and precisely record the maxillomandibular relationship. The different types of interocclusal record materials have been in use since past, none of the material were adequate enough in recording centric relation, these recoding mediums used conventionally exist few problems in transferring the working cast to the articulator like incomplete seating of the cast into the interocclusal record, improper condyle position because of loss of proprioception of the temporomandibular joint and dimensional instability of recording materials. Inaccurately recording interarch relationship and mounting casts on articulator with these records will lead to compromised occlusal relationship with lots of error, especially in complicated cases. Adequately recording and registering the centric relation is most essential step for success of any type of oral rehabilitation. ${ }^{[13][12]}$ The purpose of this review article is to evaluate the accuracy and stability of the interocclusal record materials used to record centric relation.

\section{Discussion}

Bite registrations plays an important role in achieving an accurate centric relation record that can be precisely transferred from the mouth to an articulator. ${ }^{[14][15]}$ However, when several teeth are prepared or missing, precluding a stable relationship between casts, a separate interocclusal record may be required, to precisely mount the casts, a tripod of vertical support and horizontal stability must exist between the maxillary and mandibular casts. Basically, there are two main categories of interocclusal registration: Centric interocclusal records \& Eccentric interocclusal records (Protrusive, left lateral and right lateral). ${ }^{[17]}$ The centric interocclusal records are further classified as follows: Maximum intercuspation position records: it is the interocclusal registration made at maximal intercuspation of the existing dentition. Retruded centric position records: it is the interocclusal registration made at the centric relation of the mandible. It is generally indicated when the change in the vertical dimension is to be anticipated and use of face bow is indicated in relating the maxillary model to the upper member of the articulator. The eccentric interocclusal records are further classified as follows: Lateral excursive records: the lateral excursive registration records the lateral excursive maxillomandibular relationship and is performed without occlusal contact. These records are used to set the condylar elements of an Arcon semi adjustable articulator. Protrusive interocclusal records: it registers the maxillomandibular relationship during protrusion of mandible. ${ }^{[18]}[19]$ All these records are made using various interocclusal recording medium, that should fulfil the basic requirements. Material should not displace the teeth during intercuspation; Exhibit little or no dimensional change upon setting; Accurately record the occlusal and incisal surfaces of the teeth; Remain rigid after setting; There should be minimal resistance form, Should not affect the normal closing pattern of the mandible or cause abnormal movement of the teeth during closure. The material should be biocompatible. Ease of use and modification; should not cause any adverse effects on tissues involved in the recording, finally it should be verifiable. (Pipia, 1978; Berman, 1960). Silverman (1957) stated an interocclusal record should offer no resistance to closure, have fluidity, and permit the masticatory 
mechanism to operate free from strain. A most precise centric relation can be obtained only with minimal closing force, any attempt to make a record with anything more than minimal closing force generally leads to a compromised centric relation. Various materials used as a bite registration medium are as follows. ${ }^{[20]}[21]$ The earliest practice of making interocclusal bite records were employed by using Impression plaster, commonly known as Plaster of Paris (type 1) gypsum product, which was introduced by EJ Dunning in the year 1844, later combined with modifiers to improve the physical properties of the material such as accelerate setting time and decrease setting expansion. Records made with impression plaster are accurate, rigid after setting and do not distort with extended storage, major drawback, it is difficult to manipulate as material is fluid in nature, unmanageable prior to setting, the final record obtained is brittle in nature. ${ }^{[21]}{ }^{[22]}$ Further to overcome the drawback of impression plaster the newer material Modeling compound was introduced in the year 1856 by Charle Stent, it is thermoplastic in nature, becomes rigid upon setting, has been used to fabricate interocclusal records, two potential drawbacks associated with its use include the following: difficult to record details because of high viscosity, which cause errors in repositioning on the working casts and might cause abrasion of working dies during mounting procedures. ${ }^{[2][23]}$ later in 1920's M odeling wax was introduced, the most versatile and commonly used interocclusal recording material, the reason for its versatility is its easy manipulation and uniformity, however, it is dimensionally inaccurate interocclusal recording material as it has a high coefficient of thermal expansion, that leads to distortion of wax due to release of internal stresses, thus, leading to inaccuracies in the record. ${ }^{[19][23]}$ In 1930's Zinc oxide eugenol paste was introduced by Kelly and Ward, generally used as interocclusal recording material, because of the fluidity of paste before setting, it offers minimal resistance with mandibular closure and becomes rigid after it sets finally, however, every material has its own advantages and disadvantages, the disadvantages of zinc oxide eugenol paste is that they have a prolonged setting time, significant brittleness; they stick to the teeth. As it sets by chelation reaction, the by-products formed undergoes evaporation leading to dimensional change. ${ }^{[21][23]}$ In 1961 acrylic resins were introduced by Chase to overcome the disadvantages of other interoclusal bite recording materials, most frequent application of acrylic resins as interocclusal recording medium is in the fabrication of single stop centric occlusion records. Acrylic resin is both accurate and rigid after setting, disadvantages of acrylic resin as an interocclusal registration material include dimensional instability due to continued polymerization resulting in shrinkage; rigidity of the material can damage plaster cast and dies during mounting on the articulator, acrylic resin maintains its dimensional stability during storage; hence it has been used as a bite registration material when a record is required for long term storage. Nevertheless, acrylic resin has the limitations of large polymerization shrinkage (Pattern Resin volumetric shrinkage rate: 5.07\%) and long hardening time. M ore displacements could occur in harder bite registration materials, and the error appears to be larger because of the hardening properties of acrylic resins. ${ }^{[22]}$ Elastomers were later introduced to overcome the drawbacks of acrylic resins, the elastomers being the most dimensionally stable materials till date they consistently yielded the least error among all the materials studied, they are easy to manipulate and offer little or no resistance to closure, set by addition polymerization reaction to a consistency that makes them easy to trim without distortion, and accurately reproduce occlusal details. no by-products and no loss of volatiles occur in addition silicones, accuracy is maintained with very minimal resistance to closure and easy manipulation are the main advantages of addition silicones as interocclusal recording material, however, its major disadvantage is that any compressive force exerted on these materials during mounting procedures may cause inaccuracies during mounting of the casts. Spring action found in these materials may cause inaccuracies during mounting of the casts. Thus, the records should be trimmed and carefully seated over the occlusal surface to minimize the negative 
spring action. Polyether elastomers were introduced in 1965 though it was patented in 1969. Polyether elastomeric compound was made from polyether terminated with amino groups cross linked with strong acids such as aromatic sulfonic acid esters. The cross linked rubber was reported to have high dimensional accuracy after polymerization and storage; these elastomers possess the highest recovery after deformation when compared to other elastomers. It also had the least dimensional change following removal from the mouth. The advantages of this material as an interocclusal registration material are stability and accuracy after polymerization and during storage, minimal resistance to closure and can be used without a carrier, disadvantages are that accuracy of polyether may exceed accuracy of the plaster or stone casts. Both of these factors can interfere with the placement of the cast into the recording medium during mounting procedures. The records are trimmed to remove excess material and preserve only the teeth indentations, avoiding distortions. ${ }^{[2][[23]}$

Although no material satisfies all the ideal requirements, a

\section{References}

1. M ridul thakur, veena jain, hari parkash, pravesh kumar. Journal of Indian prosthodont society.july-sept: 2012;12(3):175-181

2. Sanjay bansal, Jayant palaskar. The journal of Indian prosthodontic society. July. 2009: vol 9:issue 3

3. G S chandu, M ohd. Faisal khan, S K mishra, Pooja asnani. Evaluation and comparison of resistance to compression of various interocclusal recording media: an in vitro study journal of international oral health. $2015 ; 7(5): 24-2$

4. C sabarigirinathan, K vinayagavel, C gunasekar, Vikas, Rupkumar , Jeyanthi kumari. International journal of advancements in research \& technology.Dec.2013(2)(12),

5. Dushyant soni, Darshana shah. Interocclusal records for fixed prosthodontics: a review of various techniques. The journal of ahmedabad dental college and hospital; M arch 2011 - august 201. 2(1),

6. Balvinder singh, Divya mittal. Interocclusal records in fixed prosthodontics. International journal of advancements in research \& technology. december-2013;2(12).

7. Krishna prasad d. B. Rajendraprasad, Anupama prasad d. Divya mehra .nujhs. september 2012;2(3)

8. Fattore-bruno, ladeane, "clinical evaluation of the accuracy of interocclusal recording materials" (1979) .M aster's theses. paper 3026

9. S. Sonune, S. Dange, A. Khalikar. An accurate interocclusal record by creating a vertical stop. The journal of Indian prosthodontic society | july 2005 | vol 5 | issue 3

10. Sanjay bansal, Jayantpalaskar. Critical evaluation of various methods of recording centric jaw relation. The journal of Indian prosthodontic society | december 2008 | vol 8 | issue 4

11. Do-hyun park, Ji-man park, Jae-won choi. Un-sook kang Eun-bin bae, Young-chan jeon1. Accuracy of several implant bite registration techniques: an in-vitro pilot study. J adv prosthodont 2017;9:341-9 range of physical and chemical properties are desirable for ideal interocclusal recording material. These are low viscosity, low resistance to closure, ease manipulation, adequate working time, precision in detail, rapid hardening, bio-compatibility and verifiability of the various properties enumerated, dimensional stability and the compressive resistance characteristics are not apparent from the previous studies or from the information provided by the manufacturers ${ }^{[2]][23]}$

\section{Conclusion}

The technique chosen by the oral health practitioner to record interocclusal relations should depend on the availability of materials, experience of the operator and the clinical situation. The limitations and physical properties of all these materials include biological and manipulative errors caused by variation in the temperature between the oral cavity and the laboratory; and the errors caused by volumetric changes in the impression and stone materials. Further studies will be needed to consider these factors as an ideal interoclusal recording medium.

12. K. Karthikeyan, H. Annapurni. The journal of Indian prosthodontic society | march 2007| vol 7 | issue 1

13. Maj p dua, Gpcaptshgupta, It col s ramachandran, It col hssandhu. Evaluation of four elastomeric interocclusal recording materials. Mjafi, 2007:63(3)

14. Donald j. Pipko, Sarabjit khass. An in vitro study of the effect of different occlusal registration materials on the reproducibility of mounting casts. The journal of Indian prosthodontic society / january 2009 / vol 9 / issue 1

15. Mine dündarçömlekoglu, Muharremerhançömlekoglu. Dental occlusion. Journal of dentistry and oral biology.2017 | volume 2| issue 20 | article 111

16. Puneetbathra, Lakshmirao, Angshuman battacharya, Ritu duggal Hariprakash. Journal of Indian orthodontic society. 2002:35 vol. 113-117

17. Do-hyun park, Ji-man park, Jae-won choi1, Eun-sookkang, Eun-bin bae. Young-chanjeon, Chang-mojeong, Mi-jungyun, Jung-bohuh. Accuracy of several implant bite registration techniques: an in-vitro pilot study. The journal of advanced prosthodontics. 2017:9:341-9

18. Herbert t shillingburg, jr,dds, sumiya hobo, lowell d, richardjacob.chapter 3. Interocclusal records text book of fundamentals of fixed prosthodontics. Third edition. 1997. Pgno 35-46.

19. Sweeney s, Smith dk, Messersmith m. Comparison of 5 types of interocclusal recording materials on the accuracy of articulation of digital models. am j orthod dentofacial orthop. 2015 aug; 148(2):245-52.

20. william j. Obrien. Text book of dental materials and their selection. Fourth edition. Chapter 4. Gypsum products. Page 38-61.2002

21. william j. Obrien . Text book of dental materialsa and their selection. Fourth edition. Chapter 7. Impression materials. Page 91-113. 2002

22. Valeria D oliveria. Maria Da Gloria, A Clinical evaluation of materials for interocclusal registration in centric relation. Braz Dent J: 2000;11(1):41-47.

23. Geet Goyal, History of impression, impression materials and impression techniques in complete dentures. Journal of advanced medical ans dental sciences Research. April-june 2014.Vol 2(2). 